\title{
Creating physically accurate visual stimuli for free: Spectral rendering with RADIANCE
}

\author{
Alexa I. Ruppertsberg aNd Marina BloJ \\ University of Bradford, Bradford, England
}

\begin{abstract}
Visual psychophysicists, who study object, color, and light perception, have a demand for software that produces complex but, at the same time, physically accurate stimuli for their experiments. The number of computer graphic packages that simulate the physical interaction of light and surfaces is limited, and mostly they require the purchase of a license. RADIANCE (Ward, 1994), however, is freely available and popular in the visual perception community, making it a prime candidate. We have shown previously that RADIANCE's simulation accuracy is greatly improved when color is coded by spectra, rather than by the originally envisaged RGB triplets (Ruppertsberg \& Bloj, 2006). Here, we present a method for spectral rendering with RADIANCE to generate hyperspectral images that can be converted to XYZ images (CIE 1931 system) and then to machine-dependent RGB images. Generating $X Y Z$ stimuli has the added advantage of making stimulus images independent of display devices and, thereby, facilitating the process of reproducing results across different labs. Materials associated with this article may be downloaded from www.psychonomic.org.
\end{abstract}

The human visual system is confronted with the 2-D image of the world projected onto the retina. This complex 2-D pattern of light is the result of scene illumination, the surface reflectance properties of objects, and their spatial configuration. If we want to understand how the visual system analyzes these complex but naturalistic patterns, our stimuli need to reflect this complexity accurately; otherwise, the validity of the results may be limited. Psychophysical (Bloj, Kersten, \& Hurlbert, 1999; Gilchrist \& Jacobsen, 1984) and computational (Funt \& Drew, 1993; Nayar, Ikeuchi, \& Kanade, 1991) studies, for example, have postulated that a particular illumination phenomenon - mutual illumination-may provide cues for color and shape perception. Mutual illumination arises from light reflected between surfaces, and it has been shown that the representation of an object's color in a rendering program with three or any small number of discrete samples will lead to an underestimation of the intensity of mutual illumination (Shafer, 1992), unless all the participating surfaces and lights have flat spectra or if only one of them is not flat (see Doerschner, Boyaci, \& Maloney, 2004, for a more detailed explanation). To create accurate complex naturalistic stimuli, and not just photorealistic pictures, scientists need a rendering tool that is relatively easy to implement and, preferably, low cost.

RADIANCE (Ward, 1994), a physically based, freely available rendering package, has been used in the computer graphics, architectural, and lighting communities for some time. It is quickly becoming the image generation package of choice for the visual perception community when studying light and surface perception in complex 3-D scenes (Boyaci, Doerschner, \& Maloney, 2004; Boyaci, Maloney,
\& Hersh, 2003; Delahunt \& Brainard, 2004a, 2004b; Doerschner et al., 2004; Fleming, Dror, \& Adelson, 2003; Fleming, Torralba, \& Adelson, 2004; Yang \& Maloney, 2001; Yang \& Shevell, 2003). RADIANCE simulates the physical interaction of light and surfaces by using a backward raytracing algorithm. This means that light is followed from the point of measurement into the scene and back to the light source. However, the color properties of light and surfaces are not represented by descriptors with a physical unit, but by RGB values. On the other hand, the numerical values held in a RADIANCE image are radiance values (unit: Watt' steradian $\cdot \mathrm{m}^{2}$ ). Here, we present a method of using RADIANCE with color descriptors in physical units-namely, radiance values - to generate hyperspectral images that can be converted to $X Y Z$ images (CIE 1931 system) and then to machine-dependent RGB images. We have shown previously that this spectral-rendering method improves the simulation accuracy (Ruppertsberg \& Bloj, 2006). This article is not intended to give an introduction to RADIANCE, which can be found elsewhere (Ward Larson \& Shakespeare, 1998; www.radiance-online.org), but will focus on the implementation of spectral rendering in RADIANCE.

\section{Getting Started}

RADIANCE is an open-source code (radsite.lbl.gov/ radiance/index.html) and runs on a UNIX-based operating system (for an introduction to UNIX commands and the like, see www.ee.surrey.ac.uk/teaching/Unix/ index.html). The spectral-rendering method we present is based on shell scripts, and the image manipulation stage on MATLAB scripts under Windows. We used RADIANCE Version 3.5 on Red Hat Linux Version 8 (com- 
piled binaries for linux from the RADIANCE Web server www.radiance-online.org) and MATLAB R12.

\section{Color in RADIANCE}

Color in RADIANCE is thought to be represented by RGB triplets, which are diffuse reflectance values under an equal-energy light source (white light). If RGB values are used as color descriptors, RADIANCE will output RGB values, which can be smaller than 0 and larger than 1. To compute luminance values from RADIANCE's RGB values, the following formula is applied (Ward Larson \& Shakespeare, 1998):

$$
L=179 \cdot(0.265 \mathrm{R}+0.67 \mathrm{G}+0.065 \mathrm{~B}),
$$

which is derived from the CIE $X Y Z$-to-RGB conversion matrix based on the ideal (implemented) monitor. The CIE chromaticity coordinates for this ideal monitor's primaries and white point are

$$
\begin{aligned}
& (x, y)_{\mathrm{R}}=(0.640,0.330), \\
& (x, y)_{\mathrm{G}}=(0.290,0.600), \\
& (x, y)_{\mathrm{B}}=(0.150,0.060),
\end{aligned}
$$

and

$$
(x, y)_{\mathrm{W}}=(0.333,0.333)
$$

(see src/common/colour.h in the RADIANCE source code for more specifications of color-related numbers).

If $R G B$ values are larger than 1 or smaller than 0 , the color displayed on a monitor may have little to do with the actual calculated color of the image, since clipping will have set these values to 1 or 0 , respectively.

If, instead of RGB values, (physical) radiance values are, used as color descriptors, RADIANCE's output will be radiance values. These can be interpreted as waveband averages for the corresponding waveband (see Figure 1): The $R$ value will be the average of the red waveband (e.g., $600-700 \mathrm{~nm}$ ), the $\mathrm{G}$ value will be the average for the green waveband $(500-600 \mathrm{~nm})$, and the $B$ value will be the aver- age for the blue waveband $(400-500 \mathrm{~nm})$. If the average for the red waveband were 0.6 , for the green 0.3 , and for the blue 0.2 , the corresponding RGB-like coding [ 0.60 .3 0.2 ] would mean that the wavebands run now from 700 to 400 from left to right.

Depending on how we code color, the output of RADIANCE has to be interpreted accordingly. If we code color in RGB triplets, RADIANCE's three value per pixel output corresponds to the RGB color signal of that pixel. If we code color by radiance values, the resulting three values per pixel output from RADIANCE is interpreted as a three-waveband approximation of the color signal in radiance $\left[\mathrm{W} /\left(\mathrm{sr} \cdot \mathrm{m}^{2}\right)\right]$, as presented in Figure 1 .

\section{N-Step Rendering}

For the color descriptors based on radiance values, we implement an $N$-step algorithm. The idea behind $\mathrm{N}$-stepping is an approximation of the original signal (in our case, energy over wavelength) by $N$ steps, where $N$ can be any integer $>0$. The spectrum is divided into $N$ consecutive, equally spaced wavebands, and the average value for each of the wavebands from the original signal is calculated. Figure 2 shows a surface reflectance function (original signal) and a three-step approximation in gray. The triplet shows the average values of the three consecutive wavebands $([380-510 \mathrm{~nm}],[515-645 \mathrm{~nm}]$, and [650-780 nm]) in BGR order. These three values are then used as the color descriptor in RADIANCE - that is, [0.46 $0.270 .15]$ in RGB order (see the comment above).

For $N=9$, the spectrum is divided into nine wavebands, and nine average values are calculated (dashed line in Figure 2). To implement a nine-step approximation in RADIANCE, three images need to be rendered, each image accounting for a different part of the spectrum; that is, Image 1 will account for [380-510 nm], Image 2 for [515-645 nm], and Image 3 for [650-780 nm] (see also Figure 3).

The maximum value for $N$ is determined by the sampling resolution of the original signal. If, for example, we sampled the original signal from 380 to $780 \mathrm{~nm}$ in $5-\mathrm{nm}$

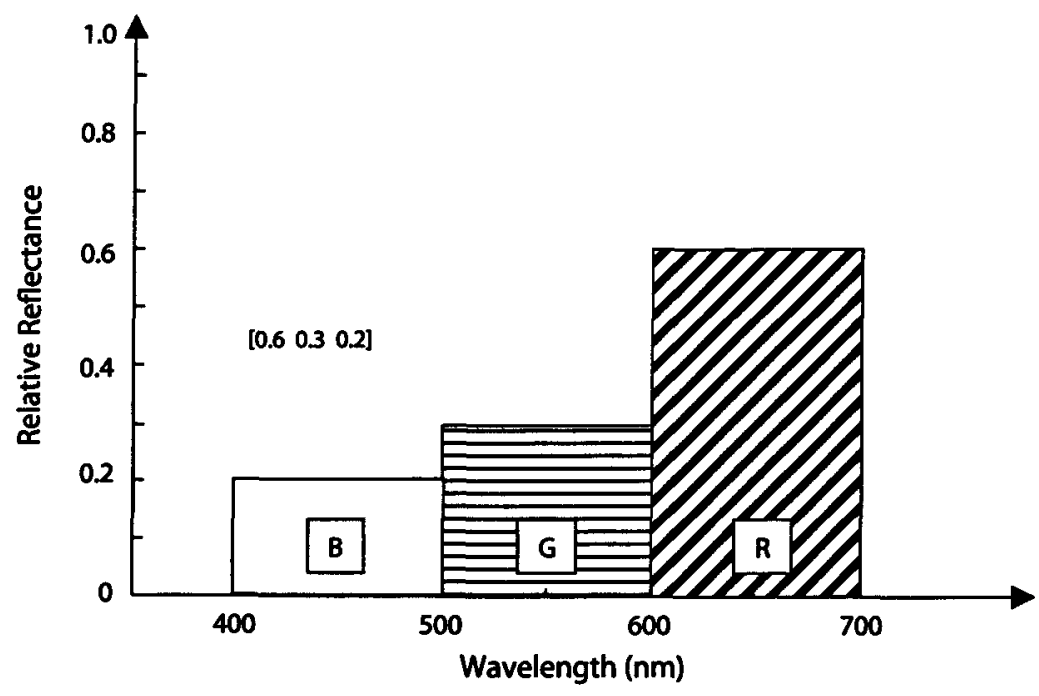

Figure 1. Interpreting RGB values as approximations of waveband averages. 


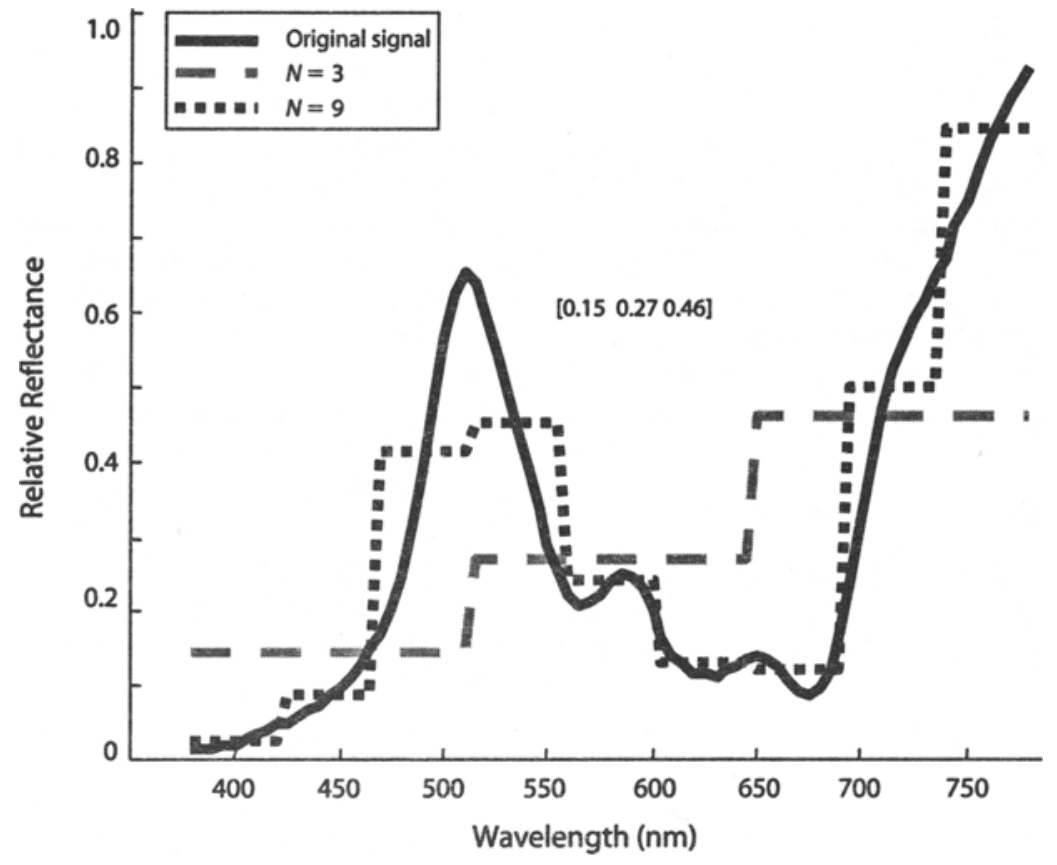

Figure 2. Example of approximating an original signal by $\boldsymbol{N}$ steps.

steps, the maximum value for $N$ would be 81 , in which case 27 images needed to be rendered for the scene.

The principle of this algorithm is related to hyperspectral imaging, in which one image is taken for each waveband, resulting in $J$ images for a scene, where $J$ is the number of wavebands. Together, these $J$ images carry the full spectral information for each single pixel in the image. To make the hyperspectral image displayable on a monitor, the information of the $J$ images has to be collapsed into a standard three-channel RGB image by convolving the spectral signature of each pixel with the $x, y$, and $z$ matching functions. This yields $X, Y$, and $Z$ tristimulus values for each pixel, which can then be converted to RGB with a conversion matrix T. T is a $3 \times 3$ matrix that depends on the monitor's primaries at maximum intensity (for a description of how to obtain this matrix, see Foley, van Dam, Feiner, \& Hughes, 1992, and Travis, 1991, among others). Multiplying the $X Y Z$ tristimulus values by $T$ yields $R G B$ values.

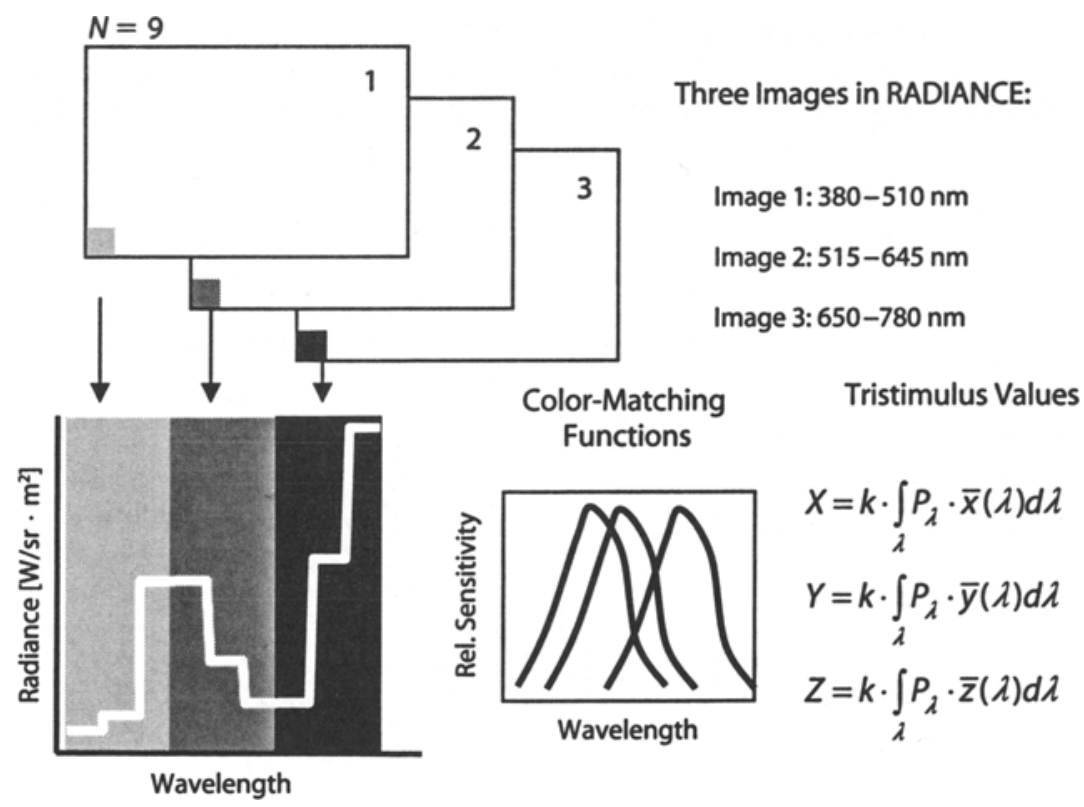

Figure 3. For $N=9$, three images are rendered, each accounting for a different part of the spectrum. By convolving the spectral signature of each pixel with color-matching functions, for example, tristimulus values can be obtained. 


\section{A Scene in RADIANCE:}

Implementation Example for $N=9$

To spectrally render a scene in RADIANCE using the $N$-step algorithm, we provide a shell script that generates binary output files, which in turn can be read by a MAT$L A B$ function and can be converted to an $X Y Z$ image. We present example files for $N=9$, but the script can easily be adapted to any required number. We assume that readers are able to generate the necessary text files.

A scene set up is described in a "* . al l" file in RADIANCE, which includes objects and light sources. Our example scene, example.all, shows a three-walled room with a floor containing a box, a ball, and a cone that is illuminated by a spotlight on the top left (see Archived Materials, below). The scene file describes the location and orientation of the objects and light sources in the scene. The object files describe the physical dimensions of the object and also contain the name of the object's color. This name has to correspond to a color name in the material files (for $N=9$, we have three material files). The material files contain the radiance values for each color and waveband; for example, if the radiance values for green for nine wavebands from 380 to $780 \mathrm{~nm}$ are [0.153540, $0.180475,0.255224$, $0.563540,0.510475,0.552224,0.0215540,0.0220475$, 0.025224], the first material file will account for the first three wavebands, [0.255224, 0.180475, 0.153540] (in RGB order); the second material file for the second three wavebands, [0.552224, $0.510475,0.563540]$; and the third material file for the last three wavebands, [0.025224, 0.0220475, 0.0215540]. See Archived Materials, below, for examples of Material Files (mat1.rad, mat2.rad, and mat3.rad).
The light source my light . rad contains the physical dimensions and parameters of the spotlight. To describe a light source in RADIANCE, we use a "* . ies" file (see chap. 8 in Ward Larson \& Shakespeare, 1998), which is converted into a "* . rad" file (with ies $2 \mathrm{rad}$ ). The color of the light source is described by providing the $-c$ parameter of the ies2rad program with three radiance values (unless it is white). As with the material files, which describe the color of the objects, we need light color files that account for the color of the light when stepping through different wavebands. For the order of values, the same logic as that for the material files applies. The light color files contain only the waveband triplets computed from the spectrum of the reflectance standard under the particular light. For $N=9$, we need three light color files. See Archived Materials for examples of Light Color Files (lightcol1, lightcol2, and lightcol3).

To spectrally render the scene with nine wavebands, the shell script "mksc" (see Archived Materials) will execute the loop three times (= number of material files). Each time it will read in a new light color file, generate a new my light . rad file, render the scene, view it from a specified viewpoint, and convert the image to a binary file and save it. The top panel in Figure 4 gives a graphical representation of the processes in mksc; fields in gray indicate files that the user has to provide. Using the call ". /mksc example," the script will generate three files called example_1 . bin, example_2.bin, and example_3.bin, For more than nine steps, more material and light color files have to be generated, and the script needs to be amended accordingly (the array "matfiles" needs to be increased). For a more detailed

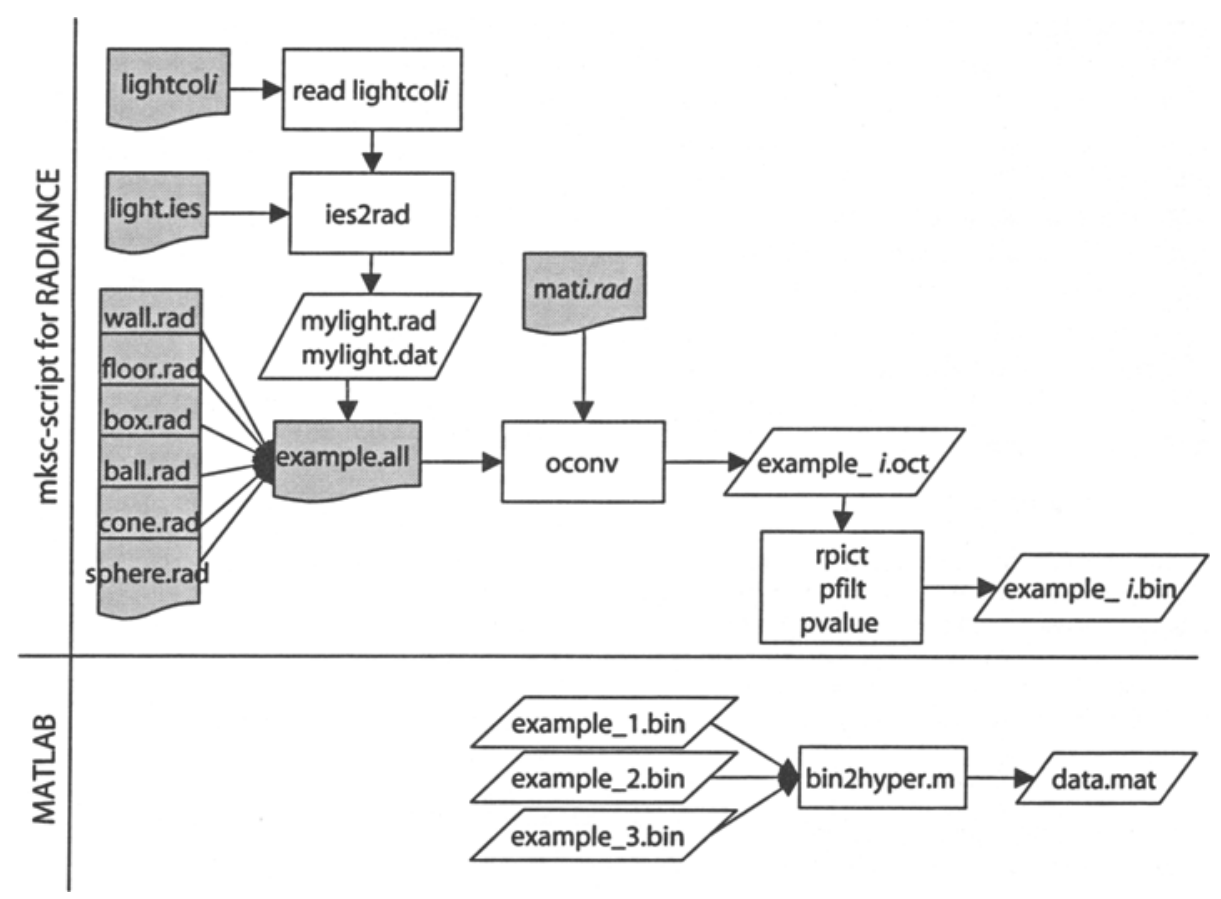

Figure 4. Graphical representation of the processes in the spectral-rendering script mksc (top panel; one iteration) and in the binary-to-hyperspectral image conversion program bin2hypex.m (bottom panel; for an $N=9$ waveband approximation). Fields in gray indicate user-provided files. 
discussion of how many wavebands are needed for obtaining good results, see Ruppertsberg and Bloj (2006).

\section{Turning Binary Files Into a Hyperspectral Image}

With a MATLAB function, bin2hyper.m (see Archived Materials; see also Figure 4, bottom panel), we can read in the binary files generated by RADIANCE and turn them into one hyperspectral image (in this case, $256 \times$ $256 \times 9$ ). By applying color matching functions, the hyperspectral image can be converted into an $X Y Z$ image $(256 \times 256 \times 3)$ and, finally, into an RGB image. Note that the resulting RGB image may contain values smaller than 0 and larger than 1 , which means that the color values are outside the range of the display device.

Although it is possible to simulate any scene in RADIANCE accurately, it does not follow that the scene can be displayed accurately on a display device. For an accurate display of the scene, the values in the scene must fall within the gamut of the display, the display must be calibrated, and, potentially, the resolution of the graphics card needs to be considered.

The method described here is similar to the one used by Yang and Maloney (2001) and it should be noted that Delahunt and Brainard's (2004b) algorithm is analogous to the one presented here. However, Delahunt and Brainard (2004b) rendered monochromatic images by setting all three channels to the same value $(R=G=B)$. Thus, for a given waveband approximation with $N$ steps, $N$ images also will be rendered, and not just $N / 3$, which makes their approach less efficient.

\section{Conclusion}

To create accurate complex naturalistic stimuli, RADIANCE, a physically based, freely available rendering package, can be implemented as a spectral-rendering program. This increases rendering accuracy, is easy to implement, and, since the software is open source, is very low cost. By giving a detailed description of the implementation and by providing example files and a shell-script to automate this process, we are confident in making this method more accessible. Generating $X Y Z$ stimuli has the added advantage of making stimulus images independent of display devices (e.g., it is well known that the output intensity of one display device diminishes over time and this, therefore, affects the conversion matrix) and, thereby, facilitating the process of reproducing results across different labs.

\section{AUTHOR NOTE}

This work was funded by the Engineering and Physical Sciences Research Council, U.K., Grant GR/S 13231 to M.B. We thank Nam Yang for helpful discussions and an explanation of his version of the $N$-step algorithm. Correspondence concerning this article should be addressed to M. Bloj, Bradford Optometry Lighting and Colour Lab, School of Life Sciences, University of Bradford, Bradford BD7 IDP, England (e-mail: m.bloj@bradford.ac.uk).

\section{REFERENCES}

Bloj, M. G., Kersten, D., \& Hurlbert, A. C. (1999). Perception of three-dimensional shape influences colour perception through mutual illumination. Nature, 402, 877-879.

Boyaci, H., Doerschner, K., \& Maloney, L. T. (2004). Perceived surface color in binocularly viewed scenes with two light sources differing in chromaticity. Journal of Vision, 4, 664-679.
BoyaCi, H., MALONEY, L. T., \& HeRSH, S. (2003). The effect of perceived surface orientation on perceived surface albedo in binocularly viewed scenes. Journal of Vision, 3, 541-553.

Delahunt, P. B., \& BraINaRD, D. H. (2004a). Color constancy under changes in reflected illumination. Journal of Vision, 4, 764-778.

Delahunt, P. B., \& BrainaRD, D. H. (2004b). Does human color constancy incorporate the statistical regularity of natural daylight? Journal of Vision, 4, 57-81.

DOERSChNER, K., BoyaCI, H., \& Maloney, L. T. (2004). Human observers compensate for secondary illumination originating in nearby chromatic surfaces. Journal of Vision, 4, 92-105.

Fleming, R. W., DroR, R. O., \& Adelson, E. H. (2003). Real-world illumination and the perception of surface reflectance properties. Journal of Vision, 3, 347-368.

Fleming, R. W., Torralba, A., \& Adelson, E. H. (2004). Specular reflections and the perception of shape. Journal of Vision, 4, 798-820.

Foley, J. D., van DAM, A., Feiner, S. K., \& Hughes, J. F. (1992). Computer gnaphics: Principles and practice (2nd ed.). Reading, MA: Addison-Wesley.

FUNT, B. V., \& DREW, M. S. (1993). Color space analysis of mutual illumination. IEEE Transactions on Pattern Analysis \& Machine Intelligence, 15, 1319-1326.

Gilchrist, A. L., \& JACOBSEN, A. (1984). Perception of lightness and illumination in a world of one reflectance. Perception, 13, 5-19.

NAYAR, S. K., IKEUCH, K., \& KANADE, T. (1991). Shape from interreflections. International Journal of Computer Vision, 6, 173-195.

RUPPERTSBERG, A. I., \& BLOJ, M. (2006). Rendering complex scenes for psychophysics with RADIANCE: How accurate can you get? Journal of the Optical Society of America A, 23, 759-768.

SHAFER, S. A. (1992). Shape recovery from interreflection. In L. B. Wolff, S. A. Shafer, \& G. E. Healey (Eds.), Physics-based vision: Principles and practice. Shape recovery (pp. 303-304). Boston: Jones \& Bartlett.

Travis, D. (1991). Effective color displays: Theory and practice. London: Academic Press.

WARD, G. J. (1994). The RADIANCE lighting simulation and rendering system. In A. Glassner (Ed.), Proceedings of the 21st Annual Conference on Computer Graphics and Interactive Techniques, SIGGRAPH 94 (pp. 459-472). New York: ACM Press.

WARD LARSON, G., \& ShakespeARe, R. (1998). Rendering with radiance: The art and science of lighting visualization. San Francisco: Morgan Kaufmann.

YANG, J. N., \& MALONEY, L. T. (2001). Illuminant cues in surface color perception: Tests of three candidate cues. Vision Research, 41, 2581-2600.

YANG, J. N., \& SHEVELL, S. K. (2003). Surface color perception under two illuminants: The second illuminant reduces color constancy. Journal of Vision, 3, 369-379.

\section{ARCHIVED MATERIALS}

The following materials associated with this article may be accessed thorugh the Psychonomic Society's Norms, Stimuli, and Data archive, www.psychonomic.org/archive.

To access these files, search the archive for this article using the journal name (Behavior Research Methods), the author's first name (Ruppertsberg) and the publication year (2008).

FILE: Ruppertsberg-BRM-2008.zip

DESCRIPTION: The compressed archive file contains 16 files:

readme.txt: an overview of the files;

example.all: a Scene file;

wall.rad, floor.rad, box.rad, ball.rad and cone.rad: Object files;

mat1.rad, mat2.rad, and mat3.rad: Material files;

light.ies: a Light Source file;

lightcol1, lightcol2, and lightcol3: Light Color files;

mksc, a shell script file to process all scene descriptions;

bin2hpyr.m: a MATLAB file to read in all bin files for an image and return the hyperspectral data.

AUTHOR's E-MAIl ADDRESs: m.bloj@bradford.ac.uk.

(Manuscript received February 26, 2007; revision accepted for publication April 25, 2007.) 